\title{
Evaluation of oxidative markers in women with invasive cervical cancer in
}

\section{Lagos, Nigeria}

\author{
Juliet O Offor ${ }^{1}$, Kehinde S Okunade², Bamidele A Iwalokun ${ }^{3}$, Ayodeji A Oluwole² and Rose I Anorlu² \\ ${ }^{1}$ Department of Obstetrics \& Gynaecology, Federal Medical Centre, Jabi, Abuja FCT, Nigeria \\ ${ }^{2}$ Department of Obstetrics \& Gynaecology, College of Medicine, University of Lagos, PMB 12003, Idi-Araba, Lagos, Nigeria \\ ${ }^{3}$ Department of Molecular Biology \& Biotechnology, Nigerian Institute of Medical Research (NIMR), Yaba, Lagos, Nigeria
}

\begin{abstract}
Epidemiological studies have showed that low levels of antioxidants induce the generation of free radicals leading to DNA damage and further mutations seen in cancer. This study evaluated the effects of oxidative markers on the occurrence and severity of cervical cancer at the Lagos University Teaching Hospital. This was an analytical crosssectional study carried out among women with histological diagnosis of invasive cervical cancer and their healthy cancer-free comparison group. Venous blood samples were collected from each participant for measurements of antioxidants (erythrocyte glutathione and vitamin C) and malondialdehyde (a marker of lipid peroxidation). Descriptive statistics were carried out for relevant demographic and clinical data. Associations between continuous variables were tested using the independent sample $t$-test or the analysis of variance for normally distributed data or the Mann-Whitney $U$ test for skewed data, whereas categorical variables were compared using the $\chi^{2}$ test. $p<0.05$ was considered statistically significant. The mean level of malondialdehyde (MDA) was statistically higher in women with cervical cancer than in their cancer-free counterparts ( $p=0.032$ ). However, the mean glutathione $(32.6 \pm 6.2$ versus $14.2 \pm 6.1 \mathrm{mg} / \mathrm{dL} ; p=0.019)$ and vitamin $\mathrm{C}$ $(12.4 \pm 2.3$ versus $14.6 \pm 2.4 \mu \mathrm{mol} / \mathrm{L} ; p=0.001)$ levels were significantly lower in the case group compared to the cancer-free group. There are statistically increasing mean levels of MDA ( $p=0.017)$ and decreasing mean levels of vitamin C ( $p=0.004)$ with increasing stages of the disease. This study showed that women with cervical cancer have low levels of antioxidants and an increased level of the oxidative marker. The levels of these markers become more pronounced as the disease progresses. This will, therefore, form the basis for the conduct of future randomised controlled trials of antioxidant supplementations among cervical cancer patients in sub-Saharan Africa.
\end{abstract}

Keywords: carcinoma, cervix, glutathione, malondialdehyde, vitamin C

\section{Introduction}

Cervical cancer is one of the challenges encountered by women as a consequence to their sexuality and childbearing potential. It is the most common female genital tract
Correspondence to: Kehinde S Okunade Email: kehindeokunade@gmail.com

ecancer 2021, 15:1266

https://doi.org/10.3332/ecancer.2021.1266

Published: 15/07/2021

Received: 22/01/2021

Publication costs for this article were supported by ecancer (UK Charity number 1176307).

Copyright: $\odot$ the authors; licensee ecancermedicalscience. This is an Open Access article distributed under the terms of the Creative Commons Attribution License (http:// creativecommons.org/licenses/by/4.0), which permits unrestricted use, distribution, and reproduction in any medium, provided the original work is properly cited. 
malignancy in most developing countries [1] and it is the second most common cancer among women [2] and it ranks fourth for both mortality and morbidity in women globally [3]. About $85 \%$ of the global burden occurs in the less developed regions, where it accounts for almost $12 \%$ of all female malignancies [2, 4]. The lifetime risk of a woman developing cervical cancer in the low-resourced setting is $2 \%-4 \%[5,6]$. About $80 \%-90 \%$ of women with cervical cancer in developing countries present with stage III and IV of the disease [7]. The primary aetiology of the disease is persistent or chronic infection with the high-risk human papillomavirus (HPV), a sexually transmitted infection [8]. However, persistent HPV infection alone is not sufficient to cause cervical cancer. Certain cofactors, such as smoking, low socio-economic status, long-term oral contraceptive use, high parity, nutritional status, co-infection with other sexually transmitted diseases, HIV and immunosuppression, have been shown to modulate the oncogenic potential of $\operatorname{HPV}[2,9,10]$. The nutritional aetiology of cervical cancer includes low dietary intake of vitamin C, carotenoids, vitamin E and folate [11]. Reactive oxygen species (ROS) generated from biochemical reactions in vitro cause lipid peroxidation products, which have been shown to induce a variety of genetic alterations, including DNA strand breaks, chromosomal abnormalities, oxidative base modifications and cellular transformation, that could result in a mutagenic lesion leading to malignant transformation [12]. The deleterious effects of these free radicals (malondialdehyde, hydrogen peroxide superoxide and nitric oxide) are counteracted by the enzymatic (superoxide dismutase, glutathione peroxidase and catalase) and non-enzymatic ( $a$-tocopherol, ascorbic acid and reduced/oxidised glutathione) antioxidants [13]. An imbalance between the production and detoxification of free radicals results in oxidative stress that damages the proteins, lipids and DNA [13]. Many epidemiology studies have further revealed that the low levels of antioxidants induce the generation of these free radicals, leading to DNA damage and further mutations [11]. Various dietary antioxidants have shown considerable promise as effective agents for cancer prevention by reducing oxidative stress which has been implicated in the development of many diseases, including cancer [14]. Vitamin C is a water-soluble antioxidant that scavenges cancer-causing free radicals such as hydrogen peroxide to prevent lipid peroxidation and destruction of cells and also neutralises carcinogenic chemicals such as nitrosamine and nitrites [15]. Glutathione provides a primary defence against oxidative stress by its ability to scavenge free radicals or participate in the reduction of hydrogen peroxide $\left(\mathrm{H}_{2} \mathrm{O}_{2}\right)$ and this is central to the detoxification of ROS [16]. Epidemiological studies have also demonstrated an association between antioxidants and cervical cancer [11, 17-19]; however, there is a paucity of studies on this subject in our environment. Moreover, the predominantly low socio-economic and poor nutritional factors could make these interactions different in our settings. This study, therefore, evaluated the role of antioxidant and oxidative markers in the occurrence and severity of invasive cervical cancer among women in Lagos, Nigeria.

\section{Patients and methods}

\section{Study design and setting}

This was an analytical cross-sectional study carried out at the Gynaecological Oncology clinic inpatient wards and the Radiotherapy Department of the Lagos University Teaching Hospital (LUTH) between August 1, 2018, and July 31, 2019. LUTH is the teaching hospital of the College of Medicine of the University of Lagos and is one of the main referral tertiary hospitals with a well-established gynaecological oncology unit in the Lagos metropolis. It is one of the centres with a functioning radiotherapy unit in Nigeria and it receives many referrals from Lagos and its surrounding states and neighbouring countries.

\section{Study population}

We recruited women with histologic-confirmed cervical cancer who were attending the gynaecological oncology units and radiotherapy clinics of LUTH (case group) and their aged-matched cancer-free women who were attending the gynaecological clinics of the hospital for non-cancerous complaints during the period of study (comparison group). Included in the study were women aged between 25 and 65 years who had not undergone any previous treatment for any cancer. Women who were athletes, smokers, alcohol consumers, those who had undergone hysterectomy, those who had other concurrent diseases such as diabetes mellitus, renal disease, liver disease, hypertension, pulmonary disease and those who withdrew consent in the course of the study were excluded. 


\section{Sample size estimation}

The minimum sample size $(N)$ was calculated using the sample size formula for comparing two means [20]:

$$
N=\frac{4 \sigma^{2}\left(Z_{c r i t}+Z_{\text {pwr }}\right)^{2}}{D^{2}}
$$

where $Z_{\text {crit }}$ is the standard normal deviate corresponding to the desired type I error rate (1.96) and $Z_{\text {pwr }}$ is the standard normal deviate corresponding to the desired statistical power of $80 \%$ (0.842). The mean vitamin C levels in cervical cancer (1.17 $\pm 0.06 \mathrm{mg} \%)$ and their control $(1.32 \pm 0.08 \mathrm{mg} \%)$ groups were obtained from the published study by Naidu et al [4], with a minimum expected difference between the two means $(D)$ being 0.15 and the standard deviation $(\sigma)$ of 0.14 . Using a $10 \%$ non-response rate, we calculated a sample size of 240 , and therefore, we recruited 120 cervical cancer cases and an equal number of cancer-free controls (with normal Pap smears) into the study.

\section{Participants' recruitment and data collection}

Eligible participants were recruited into the study using the consecutive sampling technique. A well-structured interviewer-administered questionnaire was used by the investigators to obtain participants' socio-demographic and clinical information such as age, parity, educational level, marital status, the presence of risk factors for cervical cancer and the International Federation of Gynaecology and Obstetrics (FIGO) stages for those with a histological diagnosis of invasive cervical cancer. Subsequently, $10 \mathrm{~mL}$ of venous blood samples were collected from each participant under aseptic conditions by venipuncture. A $5 \mathrm{~mL}$ aliquot was put into a plain sample bottle and the second 5 $\mathrm{mL}$ aliquot was put into a lithium heparin sample bottle. Both bottles were labelled with the participant's code and then transported to the Biochemistry and Nutrition Laboratory of the Nigerian Institute of Medical Research for analysis and measurements of serum vitamin C, glutathione (GSH) and malondialdehyde (MDA).

\section{Laboratory analyses}

Blood samples were centrifuged at 3,000 rpm for 10 minutes and immediately stored at $-20^{\circ} \mathrm{C}$ before final analysis. The prepared serum was used for vitamin $\mathrm{C}$ and MDA estimation, while the haemolysate was used for the determination of reduced GSH.

- Lipid peroxidation: MDA was estimated by measurement of thiobarbituric acid reactive substances in plasma by Niehaus and Samuelsson's [21] method. About $0.1 \mathrm{~mL}$ of the serum sample was mixed with $1.9 \mathrm{~mL}$ of the 1:1:1 ratio thiobarbituric acid (TBA)-trichloroacetic acid (TCA)-hydrogen chloride $(\mathrm{HCl})$ reagent $(0.37 \%$ TBA; $15 \% \mathrm{TCA}$; and $0.25 \mathrm{~N} \mathrm{HCl})$ making $2.0 \mathrm{~mL}$. This mixture was placed in a water bath $\left(100^{\circ} \mathrm{C}\right)$ for $15 \mathrm{~min}$, and then cooled and centrifuged at room temperature (at $1000 \mathrm{rpm}$ for 15 minutes). The absorbance of clear supernatant was measured against the reference blank at $535 \mathrm{~nm}$.

- Vitamin C: This was estimated by Roe and Kuether's method [22]. About $0.5 \mathrm{~mL}$ of the serum sample was mixed with $1.5 \mathrm{~mL}$ of $6 \%$ TCA and then centrifuged. About $0.5 \mathrm{~mL}$ volume of $0.5 \%$ oxalic acid was added to the supernatant fluid and then filtered. The filtrate was treated with $0.5 \mathrm{~mL}$ of 2,4-dinitrophenylhydrazine $(2,4 \mathrm{DNPH})$ and incubated at $37^{\circ} \mathrm{C}$ for 3 hours and then $0.1 \mathrm{~mL}$ of $85 \%$ sulphuric acid $\left(\mathrm{H}_{2} \mathrm{SO}_{4}\right)$ was added and incubated for 30 minutes. The colour developed was read at $540 \mathrm{~nm}$ against a blank solution containing $0.5 \%$ oxalic acid, 2,4 DNPH and water. The concentration of vitamin C in the sample was determined by extrapolation from the standard concentration-absorbance curve of the working standard vitamin C solution $(0.2-2 \mathrm{mg} / \mathrm{mL}$ in $0.5 \%$ oxalic acid).

- Reduce GSH: This was determined by Ellman's method [23]. One $\mathrm{mL}$ of $10 \%$ TCA was added to $1.0 \mathrm{~mL}$ homogenate and then centrifuged at $10,000 \mathrm{rpm}$ for 10 minutes. One $\mathrm{mL}$ of supernatant was treated with $0.5 \mathrm{~mL}$ of Ellman's reagent (19.8 mg of 5, 5'-dithiobis-(2nitrobenzoic acid) or DTNB in $0.1 \%$ sodium nitrate) and $3.0 \mathrm{~mL}$ of phosphate buffer $(0.2 \mathrm{M}, \mathrm{pH} 8.0)$. The absorbance was read at 412 $\mathrm{nm}$ using molar absorptivity of $1.36 \times 104 \mathrm{M} \mathrm{cm}^{-1}$. The concentration of GSH is calculated as follows: Volume sample/Total volume reaction $\times$ molar absorptivity. 


\section{Statistical analysis}

Data analyses were carried out using Epi Info Version 3.4.3 statistical software package (manufactured in 2007). Quantitative data were tested for normality with the Kolmogorov-Smirnov test with Lilliefors' significance correction. Demographic and clinical data were summarised using descriptive statistics expressed as mean (standard deviation) for continuous variables and proportion (or percentages) for categorical variables. Associations between continuous variables were tested using the independent sample $t$-test or the analysis of variance (normal distribution) or the Mann-Whitney $U$ test (skewed data), whereas categorical variables were compared using the $\chi^{2}$ test. The linear relationships between the oxidant and antioxidant markers levels were also tested and the strengths of these associations were determined using Pearson's correlation coefficient and simple linear regression. $p<0.05$ was considered statistically significant.

\section{Ethical considerations}

Ethical approval was obtained from the study hospital's Health Research Ethics Committee before participants' recruitment and ethical principles of the Helsinki Declaration were applied throughout the study. All participants were counselled before their enrolment, and they read and signed an informed consent form. The investigators ensured strict confidentiality of participant information.

\section{Results}

The overall mean age of the participants was $47.8 \pm 7.0$ years. There were statistically significant differences in parity $(p=0.002)$, age at first delivery $(p=0.001)$, educational level $(p=0.001)$ and marital status $(p=0.038)$ between the two groups of participants. There were no differences in participants' age $(p=0.170)$, body mass index (BMI) $(p=0.132)$, age at coitarche $(p=0.129)$, number of lifetime sex partners $(p=$ 0.485 ) and religion ( $p=0.141$ ) between the two groups of participants (Table 1 ).

Table 1. Baseline characteristics of the study participants $(n=240){ }^{a}$

\begin{tabular}{|c|c|c|c|}
\hline \multirow{2}{*}{ Characteristics } & Cases & Controls & \multirow{2}{*}{$p$-value } \\
\hline & $n=120$ & $n=120$ & \\
\hline Age (years) & $48.0 \pm 8.8$ & $46.0 \pm 10.7$ & 0.170 \\
\hline Median parity & $5.0(2.0-6.0)$ & $2.0(0.0-3.0)$ & 0.002 \\
\hline BMI $\left(\mathrm{kg} / \mathrm{m}^{2}\right)$ & $25.3 \pm 5.2$ & $26.4 \pm 5.1$ & 0.132 \\
\hline Age at coitarche (years) & $19.3 \pm 3.4$ & $20.0 \pm 3.0$ & 0.129 \\
\hline Age at first delivery (years) & $21.1 \pm 4.1$ & $26.7 \pm 4.5$ & 0.001 \\
\hline Median lifetime sex partners & $2.0(0.0-3.0)$ & $2.0(0.0-3.0)$ & 0.485 \\
\hline \multicolumn{3}{|l|}{ Educational level } & \multirow{5}{*}{0.001} \\
\hline Uneducated & $16(20.0)$ & $3(2.5)$ & \\
\hline Primary & $26(32.5)$ & $10(8.30$ & \\
\hline Secondary & $23(28.8)$ & $26(21.7)$ & \\
\hline Post-secondary & $15(18.8)$ & $81(67.5)$ & \\
\hline \multicolumn{3}{|l|}{ Religion } & \multirow{3}{*}{0.141} \\
\hline Christianity & $59(73.8)$ & $99(82.5)$ & \\
\hline Islam & $21(26.2)$ & $21(17.5)$ & \\
\hline
\end{tabular}


Table 1. Baseline characteristics of the study participants $(n=240) \cdot$ a (Continued)

\begin{tabular}{|l|c|c|c|}
\hline Marital status & \multirow{2}{*}{0.038} \\
\hline Single & $1(1.3)$ & $15(12.5)$ & \\
\hline Married & $54(67.5)$ & $93(77.5)$ & \\
\hline Widowed & $25(31.2)$ & $12(10.0)$ & \\
\hline
\end{tabular}

Abbreviations: BMI, body mass index (calculated as weight in kilograms divided by the square of height in meters).

aValues are given as mean $\pm \mathrm{SD}$, median (interquartile range) or number (percentage) unless indicated otherwise.

Table 2. Antioxidant and oxidant levels in patients with invasive cervical cancer and their healthy controls $(n=240)$.

\begin{tabular}{|l|c|c|c|}
\hline Biochemical markers & ICC $(\boldsymbol{n}=120)$ & Controls $(\boldsymbol{n}=120)$ & $p$-value \\
\hline MDA $(\mathrm{nmol} / \mathrm{mL})$ & $10.0 \pm 3.0$ & $4.4 \pm 1.1$ & 0.032 \\
\hline Glutathione $(\mathrm{mg} / \mathrm{dL})$ & $32.6 \pm 6.2$ & $44.2 \pm 6.1$ & 0.019 \\
\hline Vitamin C $(\mu \mathrm{mol} / \mathrm{L})$ & $12.4 \pm 2.3$ & $14.6 \pm 2.4$ & 0.001 \\
\hline
\end{tabular}

MDA, malondialdehyde; ICC, invasive cervical cancer

Table 3. Levels of antioxidants and oxidants for all the FIGO stages of cervical cancer $(n=120)$.

\begin{tabular}{|l|c|c|c|c|c|}
\hline \multirow{2}{*}{ Biochemical markers } & \multicolumn{3}{|c|}{ FIGO Stages of cervical cancer } & \multirow{2}{*}{$p$-value } \\
\cline { 2 - 6 } & Stage I & Stage II & Stage III & Stage IV & \\
\hline MDA $(\mathrm{nmol} / \mathrm{mL})$ & $7.73 \pm 1.2$ & $8.47 \pm 2.2$ & $10.74 \pm 2.4$ & $13.19 \pm 2.7$ & 0.017 \\
\hline Glutathione $(\mathrm{mg} / \mathrm{dL})$ & $35.34 \pm 5.0$ & $32.55 \pm 8.1$ & $31.69 \pm 5.7$ & $30.70 \pm 4.8$ & 0.096 \\
\hline Vitamin C $(\mu \mathrm{mol} / \mathrm{L})$ & $13.76 \pm 2.8$ & $13.49 \pm 2.5$ & $11.34 \pm 1.2$ & $10.89 \pm 0.8$ & 0.004 \\
\hline
\end{tabular}

FIGO, International Federation of Gynaecology and Obstetrics; MDA, malondialdehyde

As shown in Table 2, the mean serum MDA level was significantly higher in women with invasive cervical cancer than in their control counterparts $(10.0 \pm 3.0$ versus $4.4 \pm 1.1 \mathrm{nmol} / \mathrm{mL} ; p=0.032)$. However, the mean glutathione $(32.6 \pm 6.2 \mathrm{versus} 14.2 \pm 6.1 \mathrm{mg} / \mathrm{dL} ; p=0.019)$ and vitamin C (12.4 \pm 2.3 versus $14.6 \pm 2.4 \mu \mathrm{mol} / \mathrm{L} ; p=0.001)$ levels were significantly lower in the case group compared to the healthy control group. Further subgroup analyses among participants with invasive cervical cancer revealed increasing mean levels of MDA ( $p=0.017)$ and decreasing mean levels of vitamin C $(p=0.004)$ with increasing stage of the disease. No change in the mean level of glutathione level was observed with each FIGO stage of the disease ( $p=0.096)$ (Table 3).

In the correlation and simple regression analyses, as shown in Figure 1, we found a negative but statistically significant moderately strong association between the mean serum levels of MDA and erythrocyte GSH among women with invasive cervical cancer $(r=-0.67 ; p=0.016$ ). We also reported a negative but statistically significant moderately strong association between the mean serum levels of MDA and vitamin $C(r=-0.46 ; p=0.001)$ among these women (Figure 2).

\section{Discussion}

This study investigated the relationship between markers of oxidative stress and cervical cancer and its severity among women in Lagos. The study found a significantly higher level of serum MDA among women with cervical cancer compared to their cancer-free counterparts. We also found a statistically significant reduction in the level of serum vitamin $\mathrm{C}$ among those with invasive cervical cancer. The levels of serum 
MDA increased significantly with the stage of the disease. Inverse relationships were recorded between oxidant (MDA) and antioxidants (GSH and vitamin C) levels in the study, thus indicating a significantly high oxidative stress in participants with invasive cervical cancer.

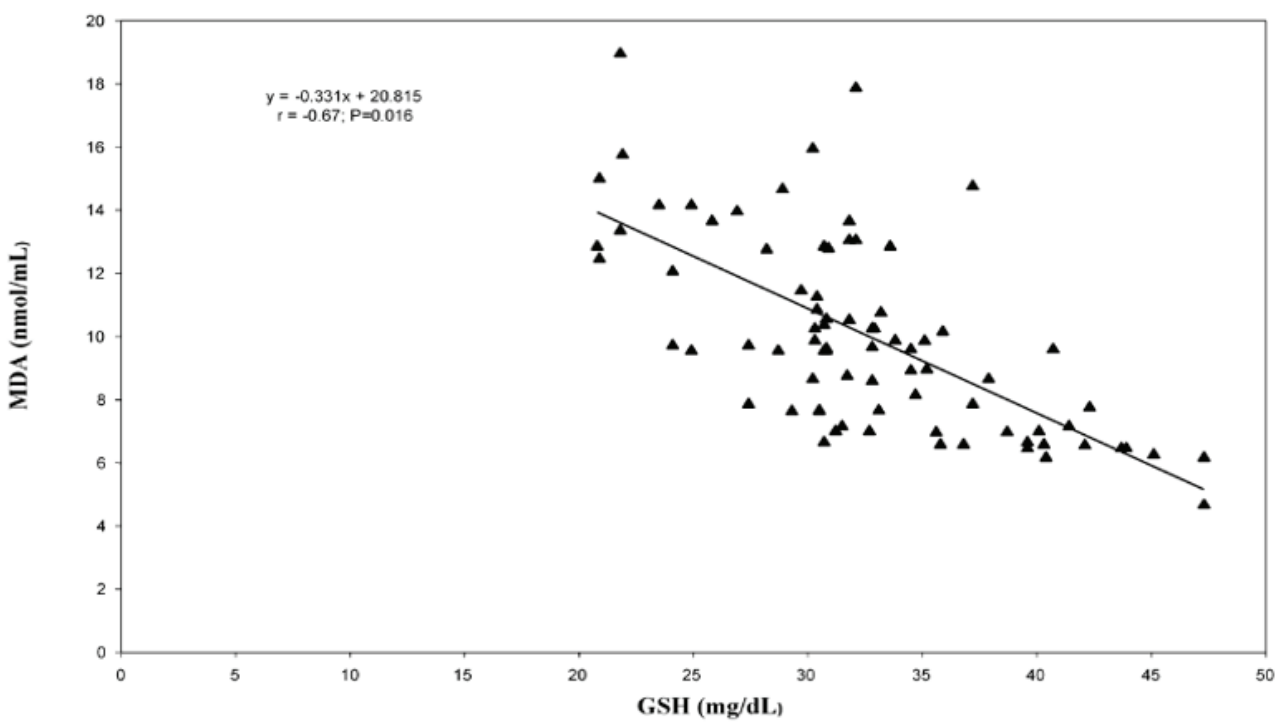

Figure 1. Relationship between erythrocyte reduced glutathione and serum malondialdehyde levels among the cancer patients.

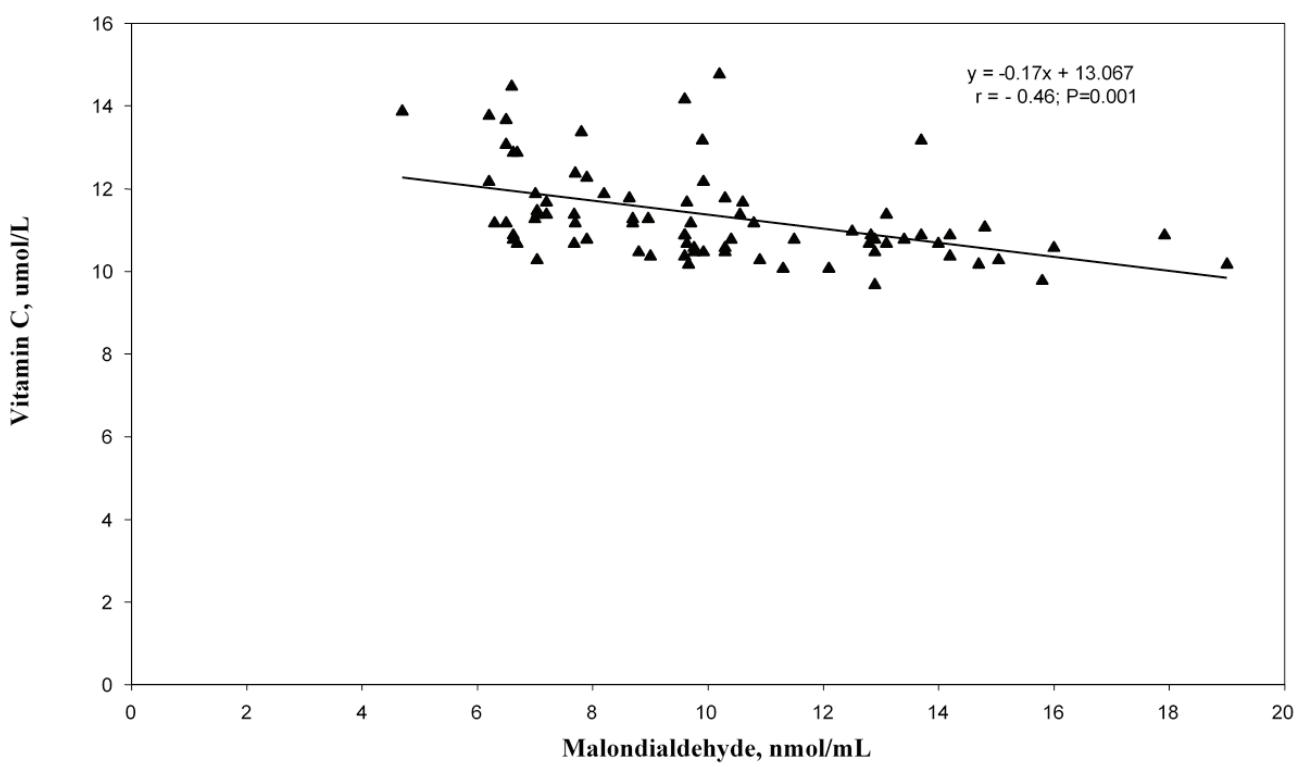

Figure 2. Relationship between serum malondialdehyde and vitamin C levels among the cervical cancer patients. 
The progressive increase in the mean MDA levels with the stage of the disease is a pointer to the excessive lipid oxidation and subsequent oxidative stress which occurs with progressive worsening of the disease. This corroborated the findings from previous studies by Naidu et al [4], Nirmala et al [24] and Beevi et al [25]. The significantly lower mean levels of erythrocyte GSH and serum vitamin C found in cervical cancer patients when compared with the cancer-free comparison group may be due to their increased use to scavenge lipid peroxides as well as their sequestration by the tumour cells $[11,13]$. This was supported by Nirmala et al [24] who observed significantly lower GSH levels in the erythrocyte and plasma of cervical cancer patients. The mean levels of serum vitamin $\mathrm{C}$ showed a statistically significant reduction with the increased stage of the disease, a finding that was in agreement with the study by Naidu et al [4] but differed from the study by Demirci et al [13], which found no significant reduction. The variation in the findings of our current study and that of Demirci et al [13] can be explained by the differences in the baseline characteristics, socio-economic status and nutritional preferences of the participants in both of these studies. The elevated oxidant and reduced antioxidants levels observed among the cervical cancer patients in this study underscore the important roles of oxidative stress in the disease progression. Beevi et al. [25] supported the hypothesis that an imbalance in the oxidant-antioxidant status culminates in lipid oxidative damage, thus potentially providing a mechanistic basis for the initiation and promotion of cancer. A major limitation of this study is the difficulty in excluding women who were unknowingly on vitamin $\mathrm{C}$ containing multivitamin supplementations which may have affected the measured serum vitamin $C$ levels in the study. The cross-sectional design of this study also made it difficult to ascribe any causality based on the associations reported. However, the study has generated hypotheses that can be tested in future wellcontrolled randomised clinical trials with a large sample size.

\section{Conclusion}

This study has shown that women with invasive cervical cancer have low levels of antioxidants (reduced GSH and vitamin C) and increased MDA, a marker of oxidative stress and these patterns become progressively pronounced with each stage of the disease. The study has, therefore, demonstrated the possible need for antioxidant supplementation in cervical cancer patients to balance their utilisation and reduce oxidative stress which causes damage to various biological molecules that leads to acceleration of the disease stage. More reliable evidence may be obtained from future randomised controlled trials of antioxidant supplementations among cervical cancer patients in Nigeria and Africa where micronutrients deficiencies are still significantly prevalent.

\section{Conflicts of interest}

The authors declared no conflict of interests.

\section{Acknowledgments}

The authors are particularly grateful to the participating women in this study. They also appreciate their colleagues in the Department of Obstetrics and Gynaecology and Radiotherapy of LUTH for their assistance in collecting data during the study.

\section{Funding declaration}

This work was supported in part by the Conquer Cancer International Innovation Grant under Project ID 16576 and the Fogarty International Centre of the National Institutes of Health under Award Numbers D43TW010934, D43TW010134 and D43TW010543. The views expressed in this publication are those of the authors and do not necessarily reflect the official views of the American Society of Clinical Oncology ${ }^{\circledR}$ or Conquer Cancer ${ }^{\circledR}$ and the National Institutes of Health. 


\section{References}

1. Kwame-Aryee R (2005) Cervical cancer Comprehensive Gynaecology in the Tropics vol 43, eds EE Emuveyan, EY Kwawukume (Accra: Graphic Packaging Limited) pp 412-428

2. WHO: International Agency for Research on Cancer (2012) Cervical Cancer: Estimated Incidence, Mortality and Prevalence Worldwide in 2012 The GLOBOCAN 2012 database (Lyon: International Agency for Research on Cancer) [Date accessed: 15/07/18]

3. Nahand JS, Vandchali NR, and Darabi H, et al (2020) Exosomal microRNAs: novel players in cervical cancer Epigenomics 12(18) 16511660 https://doi.org/10.2217/epi-2020-0026 PMID: 32957811

4. Naidu MSK, Suryakar AN, and Swami SC, et al (2007) Oxidative stress and antioxidant status in cervical cancer patients Indian J Clin Biochem 22(2) 140-144 https://doi.org/10.1007/BF02913333 PMID: 23105702 PMCID: 3453822

5. Denny L, Kuhn L, and De Souza M, et al (2005) Screen- and - treat approach for cervical cancer prevention in low-resource settings A randomized controlled trial JAMA 294(17) 2173-2181 https://doi.org/10.1001/jama.294.17.2173 PMID: 16264158

6. Mandelblatt JS, Lawrence WF, and Gaffikin L, et al (2002) Cost and benefit of different strategies to screen for cervical cancer in lessdeveloped countries J Natl Cancer Inst 44 1469-1483 https://doi.org/10.1093/jnci/94.19.1469

7. Adewole IF, Benedet JL, and Crain BT, et al (2005) Evolving a strategic approach cervical cancer control in Africa Gynecol Oncol 99 S209-S212 https://doi.org/10.1016/j.ygyno.2005.07.086 PMID: 16202445

8. World Health Organization Sexual and Reproductive Health Library (2014) Comprehensive Cervical Cancer Control; A Guide to Essential Practice vol 1, 2nd edn, (Geneva: WHO) pp 25-39 [https://apps.who.int/iris/bitstream/handle/10665/144785/9789241548953_eng. pdf;jsessionid]

9. American Cancer Society (2017) Cervical Cancer Overview (Atlanta: American Cancer Society) [www.cancer.org] Date accessed: 06/05/19

10. Kim YT, Kim JY, and Choi JS, et al (2004) Relation between deranged antioxidant system and cervical neoplasia Int J Gynecol Cancer 14 889-895 https://doi.org/10.1136/ijgc-00009577-200409000-00023 PMID: 15361200

11. Lee GJ, Chung HW, and Lee $\mathrm{KH}$, et al (2005) Antioxidant vitamins and lipid peroxidation in patients with cervical intraepithelial neoplasia J Korean Med Sci 20(2) 267-272 Date accessed: 30/04/05 https://doi.org/10.3346/jkms.2005.20.2.267 PMID: 15831999 PMCID: 2808604

12. Slaga TJ (1995) Inhibition of the induction of cancer by antioxidants Adv Exp Med Biol 369 167-174 https://doi.org/10.1007/978-14615-1957-7_15 PMID: 7598004

13. Demirci S, Zeynep O, and Celik HA, et al (2011) The interaction between antioxidant status and cervical cancer: a case-control study Tumori 97 290-295 https://doi.org/10.1177/030089161109700306 PMID: 21789005

14. Khan N, Afaq F, and Mukhtar H (2008) Cancer chemoprevention through dietary antioxidants: progress and promise Antioxid Redox Signal 10(3) 475-510 https://doi.org/10.1089/ars.2007.1740

15. Vitamin C and cancer: the irrefutable evidence (2011) [http://www.alternativehealth.co.nz/cancer/vitamins/vitc.htm] Date accessed: $08 / 09 / 11$

16. Hayes JD and Mc Lellanli (1999) Glutathione and glutathione-dependent enzymes represent a co-ordinately regulated defence against oxidative stress Free Rad Res 31 273-300 https://doi.org/10.1080/10715769900300851

17. Sekumade Al, Okunade KS, and Olorunfemi G, et al (2019) Association between serum folate level and invasive cervical cancer at a university teaching hospital in South-West Nigeria J Cancer Res Pract 6 179-183 https://doi.org/10.4103/JCRP.JCRP_24_19 
18. Kim JW, Choi EK, and Lim JH, et al (2002) Antioxidant system and oxidative stress in cervical cancer of Korean women Korean J Obstet Gynecol 45 145-151

19. Okunade KS, Dawodu OO, and Salako O, et al (2018) Comparative analysis of serum trace element levels in women with invasive cervical cancer in Lagos, Nigeria Pan Afr Med J 31194 https://doi.org/10.11604/pamj.2018.31.194.14425

20. Eng J (2003) Sample size estimation: how many individuals should be studied? Radiology 227 309-313 https://doi.org/10.1148/ radiol.2272012051 PMID: 12732691

21. Niehaus WG and Sammuelsson B (1968) Formation of malondialdehyde from phospholipids arachidonate during microsomal lipid peroxidation Eur J Biochem 6 126-130 https://doi.org/10.1111/j.1432-1033.1968.tb00428.x PMID: 4387188

22. Roe KM and Kuether CA (1943) Detection of ascorbic acid in whole blood and urine through 2, 4 DNPH derivative of dehydro ascorbic acid J Biol Chem 147 399-340 https://doi.org/10.1016/S0021-9258(18)72395-8

23. Ellman GL (1992) Tissue sulphyhydryl groups Arch Biochem Biophys 82 70-77 https://doi.org/10.1016/0003-9861(59)90090-6

24. Nirmala JG and Narendhirakannan RT (2011) Detection and genotyping of high-risk HPV and evaluation of antioxidant status in cervical carcinoma patients in Tamil Nadu State, India - a case-control study Asian Pac J Cancer Prev 12 2689-2695

25. Beevi SS, Rasheed MH, and Geetha A (2007) Evidence of oxidative and nitrosative stress in patients with cervical squamous cell carcinoma Clin Chim Acta 375 119-123 https://doi.org/10.1016/j.cca.2006.06.028 